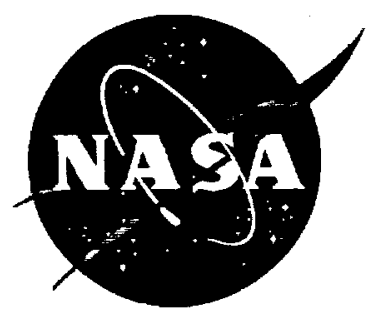

The Observational Consequences of Proton-Generated Waves At Shocks

\author{
Donald V. Reames
}

Code 661, Laboratory for High Energy Astrophysics

NASA Goddard Space Flight Center

Greenbelt, MD 20771

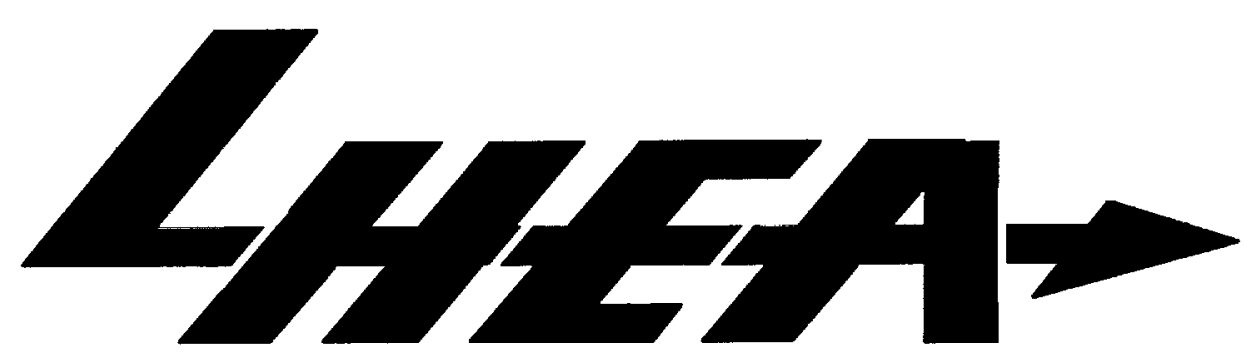

National Aeronautics and Space Administration Goddard Space Flight Center

Greenbelt, Maryland 20771 



\title{
The Observational Consequences Of Proton-Generated Waves At Shocks
}

\author{
Donald V. Reames \\ NASA Goddard Space Flight Center \\ Greenbelt, MD 20771
}

Invited paper presented at the ACE 2000 Symposium, Indian Wells, CA, Jan. 5-8, 2000 


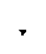

. 


\title{
The Observational Consequences Of Proton-Generated Waves At Shocks
}

\author{
Donald V. Reames \\ NASA Goddard Space Flight Center \\ Greenbelt, MD 20771
}

\begin{abstract}
In the largest solar energetic particle (SEP) events, acceleration takes place at shock waves driven out from the Sun by fast coronal mass ejections. Protons streaming away from strong shocks generate Alfvén waves that trap particles in the acceleration region, limiting outflowing intensities but increasing the efficiency of acceleration to higher energies. Early in the events, with the shock still near the Sun, intensities at $1 \mathrm{AU}$ are bounded and spectra are flattened at low energies. Elements with different charge-to-mass ratios, $Q / A$, differentially probe the wave spectra near shocks, producing abundance ratios that vary in space and time. An initial rise in $\mathrm{He} / \mathrm{H}$, while $\mathrm{Fe} / \mathrm{O}$ declines, is a typical symptom of the non-Kolmogorov wave spectra in the largest events. Strong wave generation can cause cross-field scattering near the shock and unusually rapid reduction in anisotropies even far from the shock. At the highest energies, shock spectra steepen to form a "knee." For protons, this spectral knee can vary from $\sim 10 \mathrm{MeV}$ to $\sim 1$ GeV depending on shock conditions for wave growth. In one case, the location of the knee scales approximately as $Q / A$ in the energy/nucleon spectra of other species.
\end{abstract}

\section{INTRODUCTION}

There is now a general understanding that the largest and most energetic of the solar energetic particle (SEP) events are associated with shock waves driven out from the Sun by coronal mass ejections (CMEs) (e.g. 5, 6, 7, 18). Differences between these large 'gradual' SEP events and the smaller, but more numerous, events from impulsive flares have been recently reviewed (18) and will not be discussed here.

The idea of shock acceleration of SEPs is by no means new. The first suggestion that SEP events can have either shocks or flares as progenitors was made in 1963 by Wild, Smerd, and Weiss (26) from radio observations of type II and type III bursts, respectively. However, this evidence was lost for 20 years while purveyors of the "flare myth" (5), that all particles come from flares, held sway. Difficulties in transporting SEPs across magnetic field lines over $180^{\circ}$ in solar longitude were surmounted by the mathematically contrived "coronal diffusion," which had scant basis in physics. The demise of the flare myth began when SEP ionization states were measured that were much too low for the temperatures in a solar flare $(12,24)$. At about the same time, the relationship between CMEs, shocks, and SEPs began to emerge (8).

It is well known that particles streaming out along magnetic field lines generate Alfvén waves (22) and that those waves then scatter the particles that come behind. Self-generated waves were first used as the basis of an equilibrium shock-acceleration theory for galactic cosmic rays (GCRs) by Bell (1). This theory was adapted to SEP acceleration by Lee (9). This is a seminal theory that has helped us understand many features of shock acceleration as we discuss below. However, we must remember that it is an equilibrium theory, not a dynamic theory. It does not give us realistic asymptotic behavior far from the shock or initial behavior of the first particles accelerated. In addition, Lee theory assumes a planar shock of fixed characteristics that does not describe the CME shock evolution in a nearly spherical geometry where there are substantial changes in the plasma parameters with time. In fact, Lee theory was originally applied only to locally accelerated particles; it was not well recognized that the shock, now seen at $1 \mathrm{AU}$, had been accelerating particles all the way out from the Sun. 
. 
To understand time-dependent processes in a curved geometry we must use a numerical model such as that of $\mathrm{Ng}(14,15)$. This model follows the coupled transport of particles and waves through space and time along a magnetic flux tube. At each point, the pitch-angle distribution of the particles is determined by scattering on waves, and the proton distribution function defines the growth rate of those waves.

When we discuss particle transport and scattering by waves, it is often noted that the wave turbulence, measured as field lines are convected across a magnetometer, differs from that we deduce from particle transport. This problem has been known for over 20 years (4). Energetic particles are scattered by only a small fraction of the measured magnetic turbulence. While this is often cast in dire terms as a "failure of quasi-linear theory," it is better described as a poor understanding of those wave modes that do not scatter the particles (18). Bieber et al. (2) discuss these modes in terms of two-dimensional turbulence moving across the magnetic field. Meanwhile, it is clear that energetic particles themselves are the best possible probes of that part of the turbulence that affects energetic particles.

\section{WAVES AND SHOCKS}

\section{The Streaming Limit}

It was observed 10 years ago (17) that intensities of $\mathrm{MeV}$ protons arriving early in large SEP events did not exceed a limiting value of a few hundred $\left(\mathrm{cm}^{2} \mathrm{sr} \mathrm{s}\right.$ $\mathrm{MeV})^{-1}$, as seen in Figure 1. This was understood as defining the intensity point, near the shock, where production of self-generated resonant waves decreases until the wave intensity is no longer adequate to constrain the outward streaming of the particles. From this point, particles stream outward with intensities decreasing with distance in the diverging magnetic field $(14,20)$.

The streaming limit applies only to particle transport from the source to the observer. Higher intensities can certainly exist near the source where streaming is just adequate to produce a wave-particle equilibrium. At equilibrium the intensities of both particles and waves declines in a simple way with distance from the shock (9). When shocks are sufficiently strong, these intensity peaks, called ESP events or shock spikes, survive out to $1 \mathrm{AU}$. The intensity in these peaks can rise above the streaming-

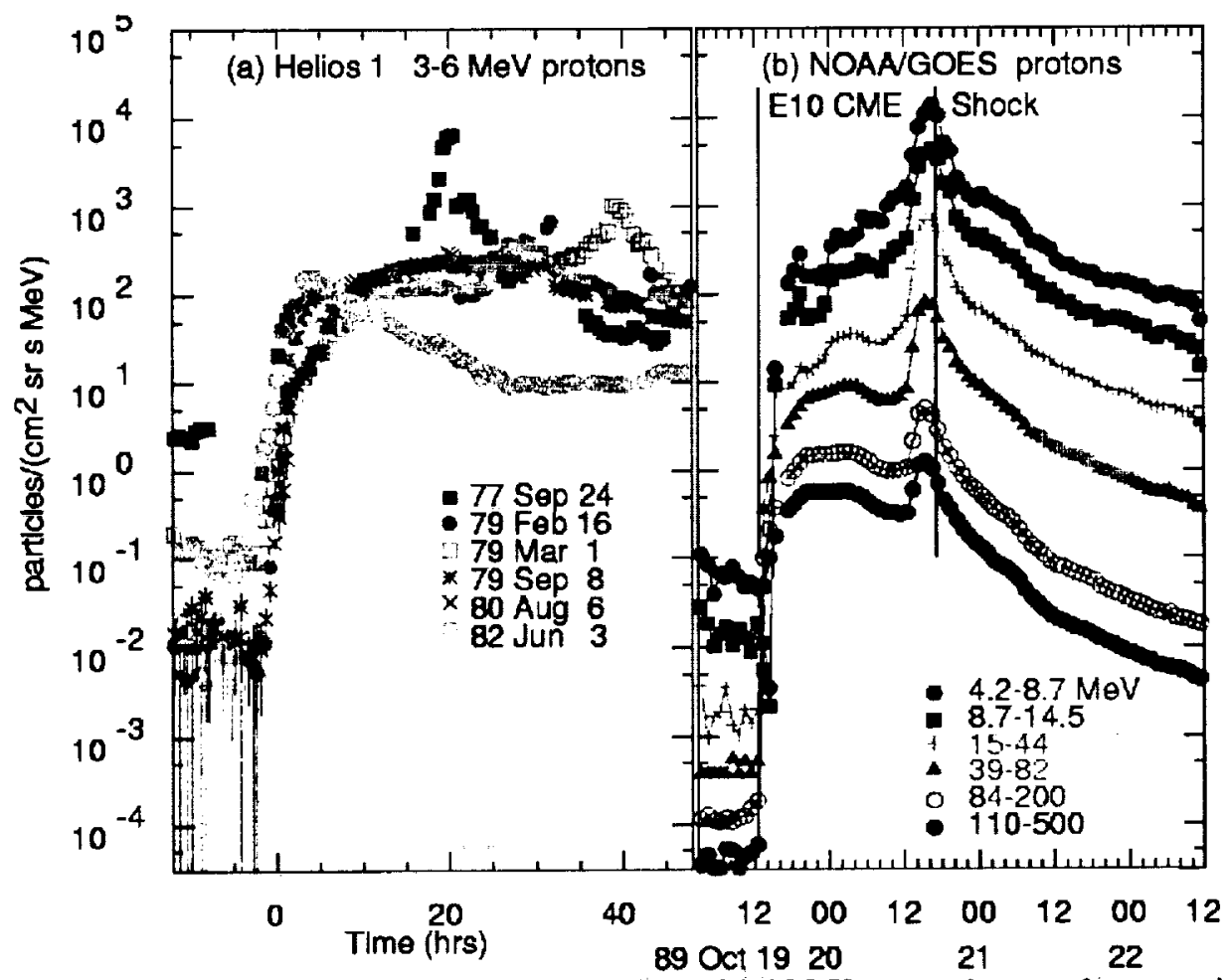

FIGURE 1. Panel (a) shows superposed intensity-time profiles of 3-6 MeV protons in several events with streaminglimited intensities early in the events. Panel (b) shows similar limits as a function of energy in the large 1989 October 19 event. Intensities often peak at the time of shock passage at values that are 10-100 times the streaming limit. 
limited value by factors of 10-100, as seen in Figure 1 . The intensity value of the streaming limit decreases with increasing energy, as seen in the right panel, Figure 1(b).

\section{Shock Acceleration}

Lee (9) theory describes the region near the shock where the particle and wave intensities are in equilibrium, i.e. it describes a planar shock peak of infinite extent after an infinite time. The theory has no temporal 'streaming limit' since the latter implies a time scale that is too short for resonant waves to grow and for equilibrium to be established. At the shock, the energy spectrum is a power law with a spectral index that depends upon the shock compression ratio. If we move a distance $x$ away from the shock, the spectrum we observe is flattened at low energies, as shown in Figure 2. It is not surprising that more resonant waves have grown at low energies where intensities are higher.

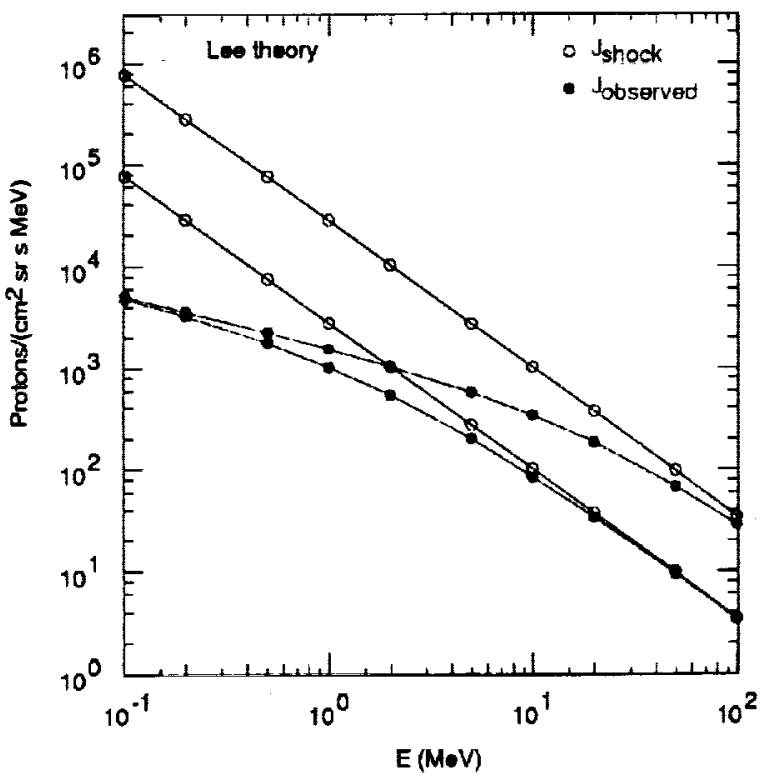

FIGURE 2. Proton intensity is shown at the shock and a flattened spectrum is seen a fixed distance away, according to Lee (9) theory. Increasing the source does not increase the intensity observed at low energy.

However, if we simply increase the normalization (injection) at the shock, Figure 2 shows that the lowenergy intensities observed at a distance $x$ are unchanged, but the flattening has extended to higher energy. This behavior is the Lee-theory's spatial equivalent of the streaming limit. The effect of the increase at the shock is only seen as an extension of the observed spectrum to higher energy. We will return to this idea again later.

It is instructive to take the simple pedestrian view of a shock shown in Figure 3. Each time particles scatter back and forth across the shock they gain an increment of velocity. Initially, just above their injection energy, they must scatter on ambient turbulence. As the particles begin to gain energy, some stream away and generate resonant Alfvén waves of wave number, $k_{\text {res }}=B / \mu P$, where $P$ is the particles rigidity and $\mu$ the cosine of its pitch angle. As later particles arrive at that rigidity, they are scattered and trapped by the resonant waves and are more likely to be accelerated further. As these moreenergetic particles stream away, they generate waves that resonate at their higher rigidity, and so forth.

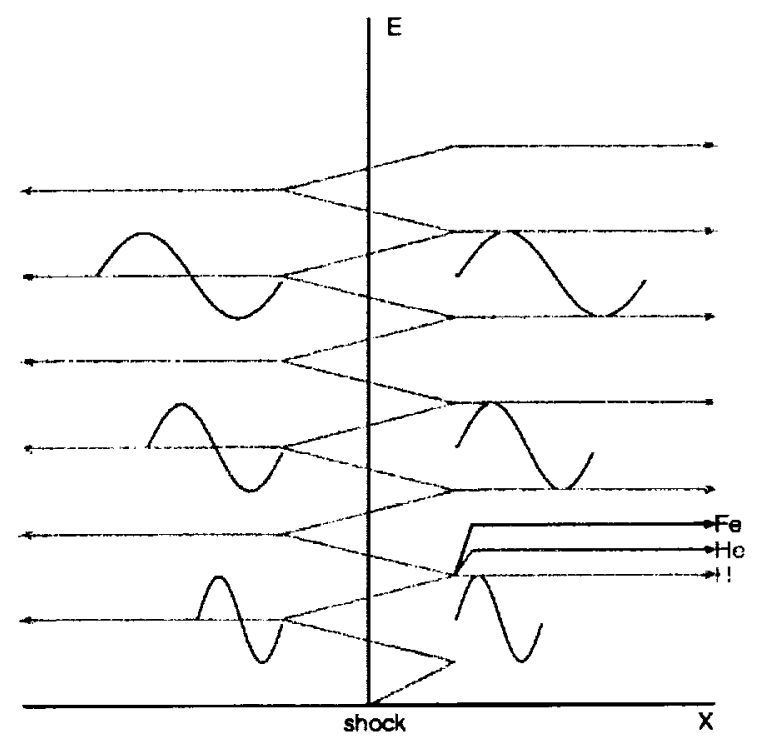

FIGURE 3. Cartoon showing trapping of particles near the shock by self-generated waves.

This process leads to the growth of a 'wall of waves' that resonates with particles of higher and higher rigidity. As equilibrium is established at each level, the intensity of escaping ions becomes fixed. Hence, as we inject more particles at the bottom, the wall must grow higher. The increase in acceleration efficiency caused by wave growth is essential for acceleration to high energies. At sufficiently high energy, where the upward flow of particles becomes inadequate to sustain the waves, a spectral 'knee' develops, and intensities plummet as particles leak easily from the shock. At the energy where intensities return to the power law in Figure 2, the real-world spectrum would actually develop a knee if there were not time to scatter and accelerate those particles. 


\section{ABUNDANCE VARIATIONS}

Most of the waves at the shock are generated by protons, the most abundant species. Other ions, accelerated to a given velocity or energy/nucleon, resonate with different wave numbers, $k$, depending inversely upon their rigidity, hence upon $Q / A$. Thus, these ions probe the shape of the wave spectrum, often producing dramatic abundance variations with time as the shock and its wave spectra evolve. To follow this evolution theoretically, we must use a numerical model such as that of $\mathrm{Ng}$ et al. (15). A comparison of observations and theory for the 1998 April 20 event is shown in Figure 4. The calculated abundances depend strongly upon the assumed values of $Q / A$ and on time variation of the shock strength, which is assumed to decrease linearly in this calculation. Considering the uncertainty in the shock evolution, the simple model captures much of the qualitative behavior of the data.

The event shown in Figure 4 is actually the largest event of the solar cycle, as measured in the fluence of $>10 \mathrm{MeV}$ protons. The associated CME is emitted from the west limb with a speed of $1600 \mathrm{~km} \mathrm{~s}^{-1}$. Because of the source longitude, it is not surprising

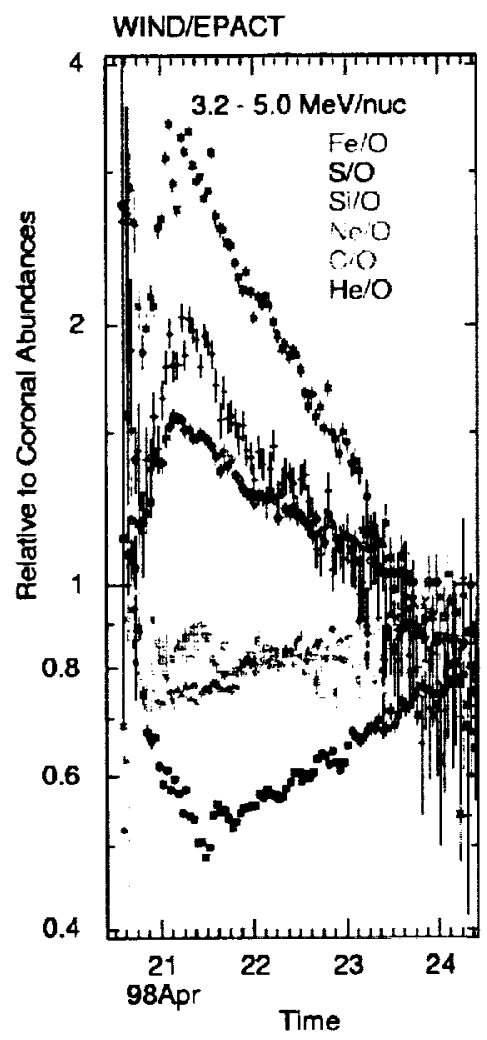

that the shock weakens considerably with time.

The abundances at any specific time result from the cumulative effects of transport through the spatially and temporally varying wave spectra from the shock to the observer. Sometimes this complexity makes it difficult to understand the physics, even when experiment and theory agree. For example, the study of large SEP events revealed a surprising feature of the initial behavior of $\mathrm{He} / \mathrm{H}$ when compared with $\mathrm{Fe} / \mathrm{O}$. Early in an event, one would expect the first particles that arrive to propagate through a pre-existing wave spectrum that might have a Kolmogorov, $\mathrm{k}^{-5 / 3}$, form. With this form, high-rigidity ions will scatter less than those of low rigidity, at the same velocity. For rising time profiles, an abundance ratio such as $\mathrm{Fe} / \mathrm{O}$ or $\mathrm{He} / \mathrm{H}$ should begin at high values and then decline with time since scattering delays ions in the denominator initially, relative to those in the numerator.

When we began to study this behavior in large SEP events, both experiment and our new theory showed the opposite initial behavior for $\mathrm{Fe} / \mathrm{O}$ and $\mathrm{He} / \mathrm{H}$ as seen in the lower right-hand panel of Figure 5 (21). We were prepared for self-generated waves with nonKolmogorov spectra, but why did they occur so early

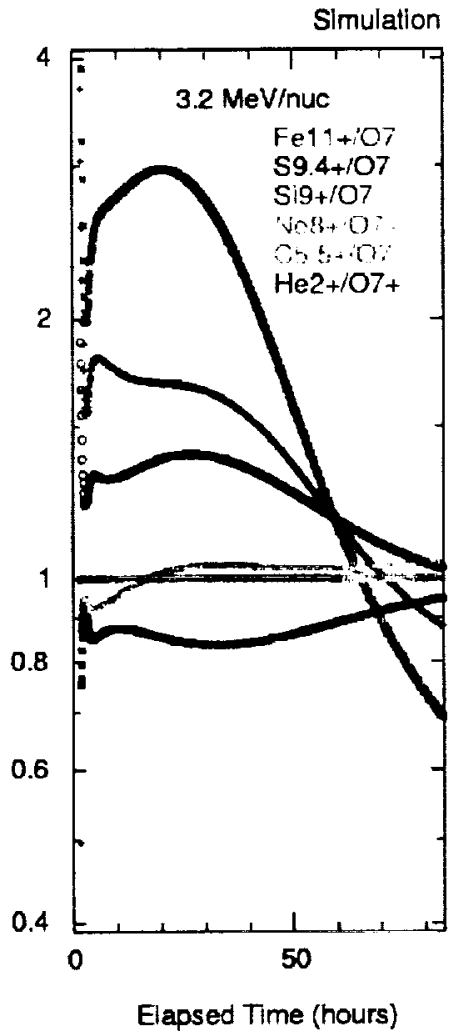

FIGURE 4. A comparison of element abundances, normalized to coronal values, in the 1998 April 20 event (25) with those calculated using the theory of $\mathrm{Ng}$ et al. (15) for specific values of the ionization states shown. 


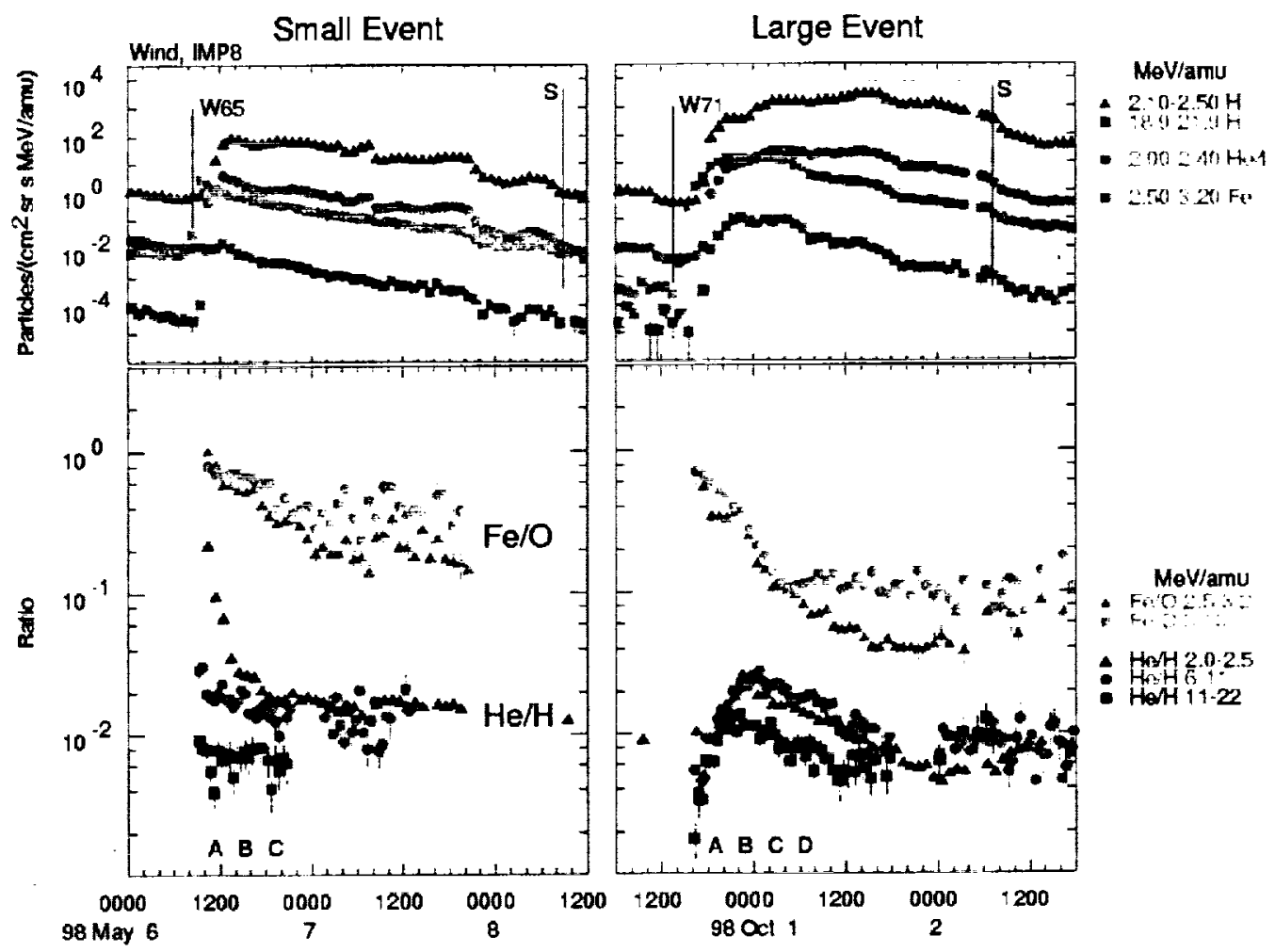

FIGURE 5. Intensities and abundances of ion species $v s$. time are compared for the small 1998 May 6 SEP event and the large 1998 September 30 event (21). The initial rise in $\mathrm{He} / \mathrm{H}$ and fall in $\mathrm{Fe} / \mathrm{O}$ are clear in the September 30 event.

in the event? In smaller events, with less wave growth, both $\mathrm{Fe} / \mathrm{O}$ and $\mathrm{He} / \mathrm{H}$ initially decline, as seen in the lower-left panel of Figure 5.

The explanation lies in the velocity of the protons generating the resonant waves $(15,21)$. The $2 \mathrm{MeV}$ protons, for example, have just arrived and are just beginning to generate resonant waves. However, the 2 $\mathrm{MeV} \mathrm{amu}^{-1} \mathrm{He}$ resonates with waves generated by protons of twice the velocity, $\sim 8 \mathrm{MeV}$, that arrived much earlier. Thus the $2 \mathrm{MeV} a \mathrm{amu}^{-1} \mathrm{He}$ can be strongly scattered when intensity of $8 \mathrm{MeV}$ protons is sufficiently high.

Figure 6 compares the proton spectral evolution for the 2 events shown in Figure 5. Spectra are taken at the times labeled by letters along the abscissa in Figure 5. Spectra in the September event are flattened at low energies at intensities we would expect from the streaming limit but they have a strong high-energy component. Spectra for the smaller May event rapidly attain power-law shapes with no excess of high-energy protons. Wave generation occurs in a shell near the shocks in both events; however, copious $2-20 \mathrm{MeV}$ protons can rapidly generate significant intensities of resonant waves all the way out to $1 \mathrm{AU}$ in the September event but not in the May event, as the spectra show.
Hints of this inverted behavior in $\mathrm{He} / \mathrm{H}$ were noticed 20 years ago $(27,13)$, but no satisfactory explanation was offered until recently $(15,21)$.

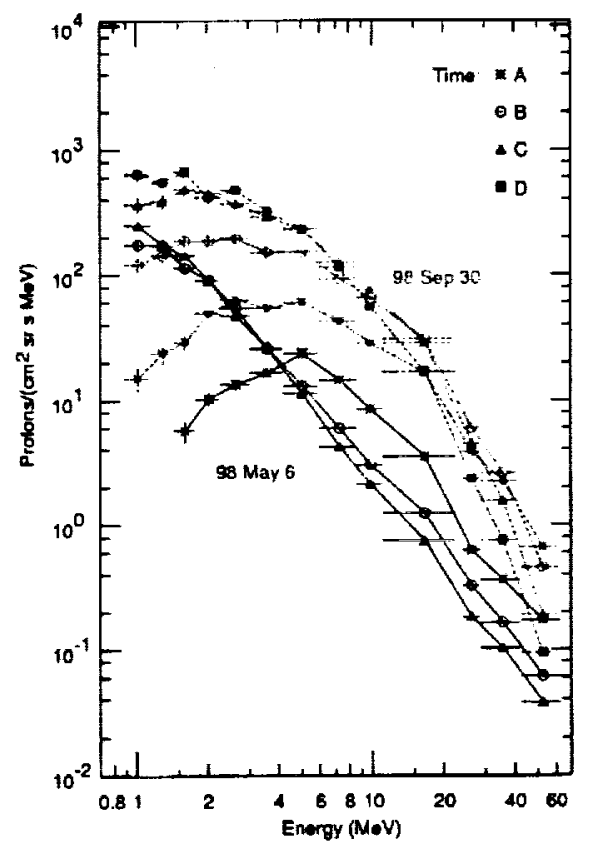

FIGURE 6. Proton spectral evolution for the two events shown in Figure $5(21)$. 


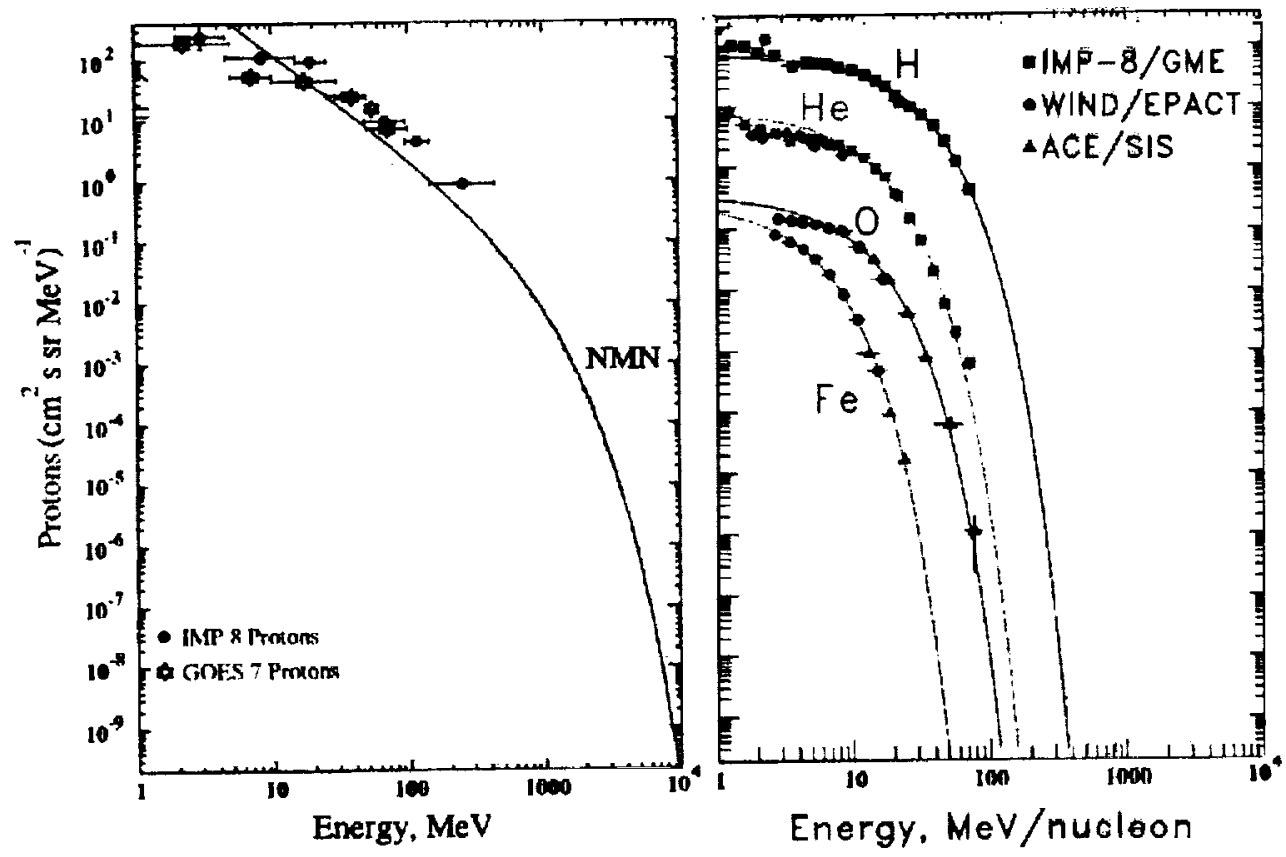

FIGURE 7. The left panel shows a spectrum from spacecraft and the neutron monitor network (NMN) in the 1989 September 30 event (11) with $E o=1 \mathrm{GeV}$. The right panel shows spectra from the 1998 April 20 event $(23)$ with Eo $=15$ $\mathrm{MeV}$.

\section{THE SPECTRAL KNEE}

We suggested earlier that acceleration would begin to fail at an energy where proton intensities could no longer sustain the growth of resonant waves. For this reason, and perhaps others, high-energy particles begin to leak from the acceleration region to form a spectral knee. The shape of the energy spectrum in this region was written as a power-law times an exponential by Ellison and Ramaty (3). Those authors examined spacecraft observations of the e-folding or "knee" energy, $E_{o}$, for protons, electrons, and He in 8 large SEP events. In 3 events they found values of $E_{o}$ of 20, 25 , and $30 \mathrm{MeV}$; in the other 5 events, they found $E_{o}$ $=\infty$, i.e. above the range of instruments. For 2 groundlevel events, spectra deduced from neutron-monitor data gave $E_{o}=5 \mathrm{GeV}$.

Data from a recent determination of the proton spectrum in the 1989 September 29 event by Lovell et al. (11) are shown in the left panel of Figure 7. This is contrasted with the more complete spectra of $\mathrm{H}, \mathrm{He}$, $\mathrm{O}$ and $\mathrm{Fe}$, in the 1998 April event recently compiled by Tylka et al. (23) for this workshop. In the first event the proton knee is at $E_{o}=1 \mathrm{GeV}$, in the second, it is at $E_{o}=15 \mathrm{MeV}$, a dramatic change for two events that are both near the west solar limb with similar CME speeds of $\sim 1800 \mathrm{~km} \mathrm{~s}^{-1}$ and $\sim 1600 \mathrm{~km} \mathrm{~s}^{-1}$, respectively.
The two events in Figure 7 actually have similar proton intensities below $-50 \mathrm{MeV}$. However, differences in the knee energies cause vastly different behavior above $\sim 100 \mathrm{MeV}$. This striking difference can have a profound application to the safety of astronauts on deep space missions, as shown in Figure 8. Soft radiation, with $E \sim 40 \mathrm{MeV}$, begins to penetrate spacecraft walls, while hard radiation, with $E>130$ $\mathrm{MeV}$, can penetrates $5 \mathrm{~cm}$ of $\mathrm{Al}$ and is difficult to shield. Behind $10 \mathrm{~g} \mathrm{~cm}^{-2}$ of material astronauts would receive a dose $-4 \mathrm{rem} \mathrm{hr}^{-1}$ at intensities in the 1989

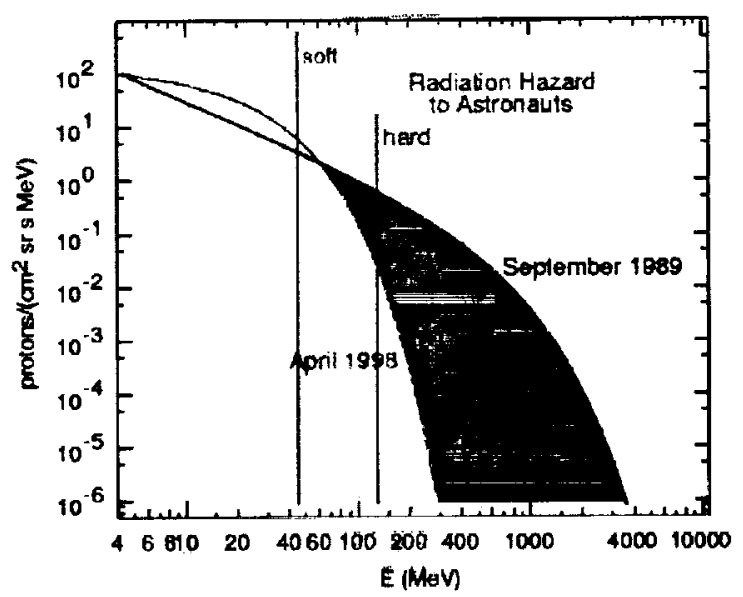

FIGURE 8. The proton spectra from Figure 7 present a drastically different radiation hazard to astronauts. 
September event, accumulating their annual dose limit of $50 \mathrm{rem}$ in relatively few hours. Differences in the knee energy alone can turn a benign event into a significant radiation hazard.

The new observations of spectra for all species in the 1998 April event show knee energies that scale like $Q / A$ throughout this event. However, knee energies seen late in the 1998 August event scale as a higher power of $Q / A$, and $E_{o}$ decreases with time (23). Unfortunately, most of the knee energies in other events of this solar cycle are above the observation range of the available instruments.

\section{DISCUSSION AND CONCLUSIONS}

Our focus on SEP events has shifted drastically in recent years. Where we once viewed event-averaged element abundances, we now look at detailed time variations in composition and spectra within an event. We see abundant evidence of wave-particle interactions, but only about half of the examples of that evidence have been included here. Other features are not presented here, such as the way SEP abundances average to coronal values, or the rate at which particle angular distributions attain isotropy in large and small events. These features were presented in an earlier review (19).

The new theory that describes the effects of waveparticle interactions $(15,16)$ does not yet fit the observations precisely (e.g. Figure 4). However, it describes the qualitative behavior of the newly observed abundance variations and it has surprised us with explanations of long-standing problems. These include the initial behavior of $\mathrm{He} / \mathrm{H}$, and differences in the angular distributions and scattering mean free paths deduced from large and small SEP events. Observational consequences of self-generated waves that have been identified (19) are the following:

1) Streaming-limited intensities early in large events $(14,17,18,20)$.

2) Flattened low-energy spectra in large events ( 8 , $16,18,25)$.

3) Systematic time variations in abundances $(16,18$, 25).

4) Abundance variations that average spatially to coronal (FIP-dependent) values $(18,19)$. (In contrast to acceleration in flares where abundances such as $\mathrm{Fe} / \mathrm{O}$ can be enhanced everywhere in space).
5) $\mathrm{He} / \mathrm{H}$ uncorrelated with $\mathrm{Fe} / \mathrm{O}$; breakdown of the power law of abundances $v s$. $Q / A$ resulting from non-Komolgorov wave spectra $(15,18,19,21)$.

6) Initial rise in $\mathrm{He} / \mathrm{H}$ in large events $(15,19,21$, 25).

7) Rapid onset of isotropy in large events, even at 1 AU (19).

8) Large variations in the energy of the spectral "knee" $(18,19)$.

A somewhat surprising aspect of these results is the importance of injection. More particles produce more resonant waves, but also, the resulting increase in efficiency causes particles to be accelerated to higher energy. When a $2000 \mathrm{~km} \mathrm{~s}^{-1}$ shock moves out from the Sun through a $500 \mathrm{~km} \mathrm{~s}^{-1}$ solar wind, ions of the bulk solar wind are injected into the shock at $-12 \mathrm{keV}$ $\mathrm{amu}^{-1}$. All species are injected at the same velocity or energy/nucleon, not the same momentum, rigidity, or energy/charge. Perhaps $\sim 1 \%$ of the ions will be successfully scattered and subsequently accelerated. As the shock speed increases, the incident flow rate and the injection velocity both increase. However, this does not fully explain why peak particle intensities are observed to increase at such a high power of the shock speed, roughly as the fourth power (19). Although, injection is clearly a nonlinear process, as we discussed in connection with Figure 3.

In large SEP events, it is no longer possible to treat the accelerated ions as test particles in the interplanetary plasma. They profoundly modify that plasma, not only near the source, but also throughout the inner heliosphere. One of the more difficult theoretical questions under investigation is the manner in which proton-generated waves are dissipated in the plasma. Intensity-dependent cascading or absorption of waves could certainly modify the particle spectra and abundances, although these effects have not yet been included in the calculations.

There will always be competing models. It is relatively easy to achieve time-varying abundances, for example, by mixing two sources with arbitrary abundances and time scales. However, the new shock models attempt to simultaneously account for intensity-time behavior, energy spectra, abundance variations and angular distributions using well-known physical processes in a single source. Where possible, parameters of the model are taken from plasma observations. The initial results are very promising. 


\section{ACKNOWLEDGMENTS}

I gratefully acknowledge the contribution made by Chee $\mathrm{Ng}$, not only to this paper, but also to my personal education during the last few years. I am also deeply indebted to Allan Tylka who has generously contributed many of his results, prior to publication, for inclusion herein. I thank Marty Lee for his comments on the manuscript.

\section{REFERENCES}

1. Bell, A. R.: 1978, Mon. Not. Roy. Astron. Soc., 182, 147.

2. Bieber, J. W., Wanner, W., and Matthaeus, W. H., J. Geophys. Res. 101, 2511 (1996).

3. Ellison, D., and Ramaty, R., Astrophys. J. 298, 400 (1995).

4. Fisk, L. A., in Solar System Plasma Physics, Vol. 1, edited by E. N. Parker, C. F. Kennel, L. J. Lanzerotti (Amsterdan: Nortb Holland), p. 177 (1979).

5. Gosling, J. T., J. Geophys. Res. 98, 18949 (1993).

6. Kahler, S. W., Ann. Rev. Astron. Astrophys. 30, 113 (1992).

7. Kahler, S. W., Astrophys. J. 428, 837 (1994).

8. Kahler, S. W., et al., J. Geophys. Res. 89, 9683 (1984).

9. Lee, M. A., J. Geophys. Res. 88, 6109 (1983).

10. Lee, M. A., in: Coronal Mass Ejections, edited by N. Crooker, J. A. Jocelyn, J. Feynman, Geophys. Monograph 99, (AGU press) p. 227 (1997).

11. Lovell, J. L., Duldig. M. L., Humble, J. E., J. Geophys. Res. 103, 23,733 (1998).
12. Luhn, A., Klecker, B., Hovestadt, D., and Möbius, E., Astrophys. J. 317, 951 (1987).

13. Mason, G. M., Gloeckler, G. and Hovestadt, D., Astrophys. J., 267, 844 (1983).

14. Ng, C. K., and Reames, D. V. Astrophys. J. 424, 1032 (1994).

15. Ng, C. K., Reames, D. V., and Tylka, A. J., Geophys. Res. Lett. 26, 2145 (1999).

16. Ng, C. K., Reames, D. V., and Tylka, A. J., Proc. $26^{\text {th }}$ ICRC (Salt Iake City) 6, 151 (1999).

17. Reames, D. V., Astrophys. J. (Letters), 358, L63 (1990).

18. Reames, D. V., Space Science Revs. 90, 413 (1999).

19. Reames, D. V. Proc. $26^{\text {th }}$ ICRC (Salt Lake City) Highlight paper, AIP Press, in press (2000).

20. Reames, D. V., and Ng, C. K., Astrophys. J. 504, 1002 (1998).

21. Reames, D. V., Ng, C. K., and Tylka, A. J., Astrophys. J. (Letters) 531, L83 (2000).

22. Stix, T. H., The Theory of Plasma Waves (New York: McGraw-Hill) (1962).

23. Tylka, A. J., Boberg, P. R., McGuire, R. E., Ng, C. K., and Reames, D. V., ACE 2000 Workshop, this volume.

24. Tylka, A. J., Boberg, P. R., Adams, J. H., Jr., Beahm, L. P., Dietrich, W. F., and Kleis, T., Astrophys. J. (Letters) 444, L109 (1995)

25. Tylka, A. J., Reames, D. V., and Ng, C. K., Geophys. Res. Lett. 26, 145 (1999).

26. Wild, J.P., Smerd, S. F., and Weiss, A. A., Ann. Rev. Astron. Astrophys. 1, 291 (1963).

27. Witte, M., Wibberenz, G., Kunow, H., and MullerMellin, R., Proc. $16^{\text {th }}$ ICRC, (Kyoto) 5, 79, (1979). 
